\title{
The Effects of Oral Consumption of Selenium Nanoparticles on Chemotactic and Respiratory Burst Activities of Neutrophils in Comparison with Sodium Selenite in Sheep
}

\author{
Gholam Ali Kojouri • Sirous Sadeghian • \\ Abdonnaser Mohebbi • \\ Mohammad Reza Mokhber Dezfouli
}

Received: 9 September 2011 / Accepted: 17 October 2011 / Published online: 22 November 2011

(C) The Author(s) 2011. This article is published with open access at Springerlink.com

\begin{abstract}
The present study was designed to compare the effects of nano-selenium and of sodium selenite on the chemotactic and respiratory burst activities of neutrophils in sheep. Fifteen sheep were randomly divided into three groups. Groups 1 and 2 received selenium nanoparticles $(1 \mathrm{mg} / \mathrm{kg})$ or sodium selenite $(1 \mathrm{mg} / \mathrm{kg})$ orally, respectively, for ten consecutive days, and the third group was considered as the control. To determine the chemotactic and respiratory burst activities of the neutrophils, the leading front assay and the NBT test were used on heparinized blood samples that were collected at different intervals (days 0,10 th, 20th, and 30th). The results obtained showed that the chemotactic activities in groups 1 and 2 increased significantly on the 10th, 20th, and 30th day, compared to day 0 , and on the 20th day in comparison with the 10th day, while in group 2 , there was a significant decrease on the 30th day compared to the 20th day. The chemotactic activities in group 1 were significantly higher than in group 2 on the 10th day and in the control group on the 10th, 20th, and 30th day, but the chemotactic activities
\end{abstract}

\section{G. A. Kojouri · A. Mohebbi}

Department of Clinical Science, Faculty of Veterinary Medicine, University of Shahrekord,

Shahrekord, Iran

S. Sadeghian $(\bowtie) \cdot$ M. R. Mokhber Dezfouli

Department of Clinical Science, Faculty of Veterinary Medicine,

University of Tehran,

Tehran, Iran

e-mail: dr.sirous.sadeghian@gmail.com in group 2 were significantly higher than those in the control group only on the 20th day. On the 30th day into the experiment, the respiratory bursts in groups 1 and 2 were significantly stronger in comparison with those at day 0. Overall, nano-selenium increased the chemotactic and respiratory burst activities more significantly than sodium selenite, which is suggestive of a stronger stimulatory effect of the Se nanoparticles on intracellular activities.

Keywords Nano-selenium - Sodium selenite - Chemotaxis · Respiratory burst $\cdot$ Sheep

\section{Introduction}

Selenium ( $\mathrm{Se}$ ) is an essential micronutrient for higher animals and humans [1, 2]. The best-known biological roles of selenium are linked to its presence as the functional component in selenoenzymes such as glutathione peroxidase (GPx) and thioredoxin reductase (TrxR). Se incorporates into proteins such as selenocysteine and prevents oxidative damage to body tissues [3]. It plays important roles in antioxidant defensive system, regulation of thyroid hormones metabolism, and cell growth [4]. The cancer preventive properties of Se have been proved according to epidemiologic, clinical, and experimental studies [5]. The antioxidant and prooxidant effects of $\mathrm{Se}$ as well as its bioavailability and toxicity depend on its chemical form. Studies have demonstrated that Se nanoparticles are more efficient than selenite, selenomethionine, and methylsele- 
nocysteine in upregulating selenoenzymes in mice and rats, while exhibiting significantly lower acute toxicity [6-9]. Nano-Se can be used as an antioxidant with reduced risk of Se toxicity and kind of chemopreventive agent because the induction of GST by Se is a crucial mechanism for this chemopreventive effect [6]. Nano-Se in different sizes (5$200 \mathrm{~nm}$ ) has equal capacity for the induction of selenoenzymes in cell culture and in mice [10], although another study showed that different sizes of nano-Se had different potency in direct prevention of free radicals in vitro [11]. Among the nutritional factors that affect the chemotaxis, phagocytosis, and the respiratory burst of the neutrophils' activities under physiological and pathological conditions, selenium is very important [12]. Adequate levels of selenium in the feed prevent the accumulation of lipid hydroperoxides in organs and tissues and protect them from damage by oxygen radical species [13-15]. Accordingly, the present study was designed to compare the chemotactic and respiratory burst activities of neutrophils in healthy sheep receiving selenium either in the form of nanoparticles or as sodium selenite, applying the leading front and nitroblue tetrazolium (NBT) assay.

\section{Materials and Methods}

\section{Animals}

Fifteen healthy, 5 to 12 months old, Lori-Bakhtiari sheep were selected and randomly divided into three groups. The sheep were orally given nano red selenium (group I; $1 \mathrm{mg}$ / $\mathrm{kg} \mathrm{B.W.)} \mathrm{and} \mathrm{sodium} \mathrm{selenite} \mathrm{(group} \mathrm{II;} 1 \mathrm{mg} / \mathrm{kg} \mathrm{B.W}$.) for ten consecutive days. The control group was given distilled water (group III).

\section{Ration}

During the study period, sheep were fed alfalfa hay and barely. The mean value of Se (mean $\pm \mathrm{SE}$ ) in the diet on dry matter basis was $0.49 \pm 0.15 \mathrm{mg} / \mathrm{kg}$.

\section{Nano Red Selenium Preparation}

Nano red elemental selenium particles (Nano-Se) were synthesized as described previously by Zhang et al. [10]. The material includes $\mathrm{SeO}_{2}$ and ascorbic acid. The ascorbic acid solution was added into the aqueous solution of $\mathrm{SeO}_{2}$ to initiate the reaction. After the addition of ascorbic acid, nano red selenium particles began to form, causing the solutions to change from colorless to red. The red color of the ascorbic acid-treated solutions suggested that the selenium was present either in its amorphous or monoclinic forms [10]. The sizes of the obtained nano-Se particles ranged from 80 to $200 \mathrm{~nm}$ as determined by scanning electron microscopy.

\section{Sampling}

Blood samples ( $20 \mathrm{ml}$ of each sheep) were collected from the jugular veins at the beginning of the experiment (day 0), and subsequently on days 10,20 , and 30 . The samples were put in acid-washed polyethylene tubes containing heparin as the anticoagulation agent.

\section{Chemotactic Activity Measuring Assay}

\section{Isolation of the Neutrophils}

The neutrophils were isolated immediately after sampling in each day, and the $20 \mathrm{ml}$ of heparinized blood was centrifuged at $1,000 \times g$ and temperature $4^{\circ} \mathrm{C}$ for $15 \mathrm{~min}$. Then, the buffy coat layer was isolated, and $2 \mathrm{ml}$ of phycol was added to it from top to bottom: (1) the leukocyte-monocyte layer, (2) the neutrophil layer, and (3) the RBC sedimentation layer.

The upper fluid was removed, and the neutrophil layer was collected slowly with a sampler and added to $2 \mathrm{ml}$ of RPMI 1640. To ascertain the purity of the neutrophil samples, smears were made on slides, and then the slides were stained by Giemsa method and surveyed with microscope (mean, three slides).

\section{LPS Preparation}

In this study, Escherichia coli K-12 bacteria lipopolysaccharide (LPS) were used as obtained from Sigma, catalog number B: BVDApo1B15. The LPS dilution was 1:5,000 using RPMI 1640 as the diluent.

\section{Leading Front Test}

For the exact performance of this test, all the instruments used were sterilized, and all stages of the assay were done in a biological hood under laminar air flow conditions. At the beginning, two to three sterile Whatman papers were placed at the bottom of six house plates, and for each sample, two houses were considered (one house as negative control and another as positive control). The negative control houses contained $1.5 \mathrm{ml}$ of RPMI 1640 and $150 \mu \mathrm{l}$ of FCS, and the positive control houses contained $.5 \mathrm{ml}$ of RPMI 1640, $150 \mu \mathrm{l}$ of FCS, and 100-150 landa of LPS (At the time of RPMI 1640 preparation, penicillin and streptomycin antibiotics were added). For each sample, two Boyden chambers were considered, and the neutrophil solution prepared last was added to the chambers so that their bottoms are convex. A 3$\mu \mathrm{m}$ micropore filter was then placed on each chamber, and they were put on the negative control houses invertedly in 
order to prevent the environments from becoming dry. Sterile distilled water was poured between the plate houses, and the plates were put in a $37^{\circ} \mathrm{C}$ incubator for $3 \mathrm{~h}$. During this period, the neutrophils moved through the micropore filter holes towards the chemotactic material (LPS). The plates exited from the incubator after $3 \mathrm{~h}$, and the stages of fixation and staining were done.

\section{Fixation and Staining}

In this stage, each micropore filter containing neutrophils was fixed and stained as follows: first, the filters were placed in isopropanole for $20 \mathrm{~min}$ so that the fixation process could be completed. The filters were then placed in sterile distilled water for 2-3 min until isopropanole was removed from the filters inside. The filters were placed in hematoxylin solution, methylene blue, and isopropanole for 2-3 min in each stage, respectively. In the final stage, the micropore filters were placed in xylol for 2-3 min and were surveyed with a microscope after drying.

\section{Neutrophils' Motility Measuring Assay}

The filters were placed on slides so that their surface was facing upward. To make the filters more transparent, a xylol droplet was poured on the slide, and lens number 40 and 100 were used to focus on the highest level of the filter (where the most cell accumulation was observed). In this condition, the number on the microscope micrometer screw was noted, and the screw was moved slowly until the filter down layers appeared; the action was continued till the stained cells inside the filter were observed. The last place where the neutrophils were observed was considered to be the maximum limit of migration, and the number on the micrometer screw was noted. Subtraction of the first and second number showed the distance traveled by the neutrophils. For each sample, five places in different points of the filter were observed randomly by two microscope lenses, and the average was registered as the distance traveled by neutrophils.

\section{Respiratory Burst Measuring Assay}

For respiratory burst assay, NBT reduction test was used [16] in which the oxidant substances inside the phagocytic cells were reduced by nitroblue tetrazolium and changed color.

\section{NBT Solution Preparation}

Five milligrams of NBT yellow powder, $85 \mathrm{mg}$ of BSA powder, and $5 \mathrm{ml}$ of PBS $1 \times$ buffer were mixed and shaken slowly so that the BSA and NBT were solved in the buffer. Before the beginning of the process, $100 \mu \mathrm{l}$ of PMA $(20 \mathrm{Mg} / \mathrm{ml})$ was added to the complex as motivator [17].

\section{Wright-Geimsa Buffer Preparation}

For this purpose, $2 \mathrm{~g}$ of $\mathrm{K}_{2} \mathrm{HPO}_{4}$ was mixed with $1 \mathrm{~g}$ $\mathrm{KH}_{2} \mathrm{PO}_{4}$, and the mixture volume was extended to $1,000 \mathrm{ml}$ using distilled water, and the solution $\mathrm{PH}$ was regulated by a PH meter which had to be between 6.9 and 7.2.

\section{NBT Test Method}

Fifty landa of heparinized blood was put in the microtube, and 50 landa of NBT solution was added to it; the microtube was shaken by the shaker carefully, and the microtubes were placed at $37^{\circ} \mathrm{C}$ bain-marie for $30 \mathrm{~min}$. The microtubes were then centrifuged at $1,500 \times \mathrm{g}$ for $3 \mathrm{~min}$. The suspension on each sample was removed by a sampler value of 50 landa, and a smear was made of the cells remaining at the bottom of the microtube on slide. Three slides were made for each sample. After the smears had dried, Wright-Geimsa color was poured on the slides, and after 2-3 min, Wright-Geimsa buffer was added onto the slides. Slides were washed with water after $4 \mathrm{~min}$, and the color remaining on the slides was cleared with alcohol. In the next stage, 100 neutrophils of each slide were counted, and the percentage of the cells which had reduced NBT was determined (colored blue spots were observed on cytoplasm). Eventually, the average of the percentages obtained from the slides related to each sample was considered as the percentage of neutrophils having reduced NBT $[18,19]$.

\section{Statistical Analysis}

The results were statistically analyzed by Sigma State (the second version) program and one-way analysis of variance tests. Also, Tukey test and Dunnett's method were used for further evaluations at the level $P<0.05$.

\section{Results}

\section{Chemotactic Activity}

The results of neutrophils' chemotactic activity value in each group in different days of sampling are presented in Table 1. It should be noted that there were no significant differences in the chemotactic activity values of the three groups at the beginning of the experiment (day 0). In group 1 (nano-selenium), on the 10th and 20th day, the values of chemotactic activity increased to $39.860 \pm 5.956$ and $51.440 \pm 7.292$, respectively, $(P<0.05)$, in comparison with the basal level (32.120 \pm 1.303$)$. The statistical survey showed that there was a significant increase in chemotactic activity on the 10th, 20th, and 30th day in comparison with day 0 ( $P$ value was $0.035,<0.001$, and 0.036 , respectively). Also, the chemotactic activity on the 20th day was 
Table 1 Changes (mean \pm SD) in the chemotactic activity of each of the groups during sampling at different days

\begin{tabular}{llll}
\hline Group sampling days & 1 (nano-selenium) & 2 (sodium selenite) & Control \\
\hline 0 & $32.120 \pm 1.303$ & $30.680 \pm 0.858$ & $32.240 \pm 1.781$ \\
10 & $39.860 \pm 5.956^{* * * * * *, * * * * *}$ & $32.340 \pm 1.361^{*}$ & $33.500 \pm 0.949$ \\
20 & $51.440 \pm 7.292^{* * * *, * * * *}$ & $59.480 \pm 11.146^{* * * *, * * * *}$ & $34.234 \pm 2.035$ \\
30 & $42.060 \pm 7.997 *, * * * *$ & $41.300 \pm 7.772 *, * * *$ & $32.700 \pm 4.176$ \\
$P$ value & $P<0.05$ & $P \leq 0.05$ & $P>0.05$ \\
\hline
\end{tabular}

${ }^{*} P<0.05$ (significant increase on day 0 ); $* * P<0.05$ (significant increase on the 10 th day); ${ }^{* * *} P<0.05$ (significant reduction on the 20th day); $* * * * P<0.05$ (significant increase to the control group); $* * * * * P<0.05$ (significant increase to group 2 (sodium selenite)

significantly more than on the 10th day $(P=0.025)$. As Table 1 clearly shows, in group 1 , the chemotactic activity decreased from the 20th day on, but statistically, there were no significant differences between the 20th and 30th day $(P>$ 0.05 ). The statistical survey in group 2 (sodium selenite) showed that there was a significant increase on the 10th, 20th, and 30th day in comparison to the day 0 ( $P$ value was $0.050,<0.001$, and 0.016 , respectively). A significant increase on the 20th day in comparison with the 10th day $(P<0.001)$ was also seen. Unlike group 1 , in group 2 , the decreasing chemotactic activity on the 30th day in comparison with the 20th day was statistically significant $(P=0.017)$. In the control group, there were no significant differences in the chemotactic activity value on any of the sampling days, neither with the basal level (day 0) nor with each other. By comparing the means of the three groups together, it was determined that there were no significant differences in chemotactic activity value on the day $0(P>0.05)$. The chemotactic activity value in group 1 showed a significant increase on the 10th, 20th, and 30th day in comparison with the control group ( $P$ value was $0.004,0.001$, and 0.049 , respectively) and on the 10th day compared with group 2 $(P=0.025)$, whereas in group 2 , there was a significant increase in the chemotactic activity value just on the 20th day in comparison with the control group $(P=0.001)$.

\section{Respiratory Burst Activity}

The results of the neutrophil value, which reduced NBT in each of the groups in different days of sampling, are presented in Table 2. It should be noted that there were no significant differences in respiratory burst activity values of the three groups at the beginning of the experiment (day 0), on the 10th, and on the 20th day. In group1 (nano-selenium), and from days 0 to 30 , respiratory burst activity values increased from $11.174 \pm 6.568$ to $21.322 \pm 7.316$. The statistical survey showed that there were no significant differences on the 10th and 20th day in comparison to day 0 , and the 10th day in comparison with the 20th day. Also, there were no significant differences on the 30th day in comparison with the 10th and 20th day, but a significant increase in respiratory burst activity value was observed on the 30th day compared to the day $0(P \leq 0.050)$. These results show that respiratory burst activity increases as time passes so that most activity occurred on the 30th day. In group 2 (sodium selenite), as with group 1 , there were no significant differences on the 10th and 20th day together and in comparison with the days 0 and 30 , but a significant increase in respiratory burst activity value was observed on the 30th day in comparison to the day $0(P=0.032)$. In the control group, there was no significant difference in respiratory burst activity value in any of the sampling days, either with the basal level (day 0) or between each other. However, after oral consumption of selenium admixtures on the 10th, 20th, and 30th day, respiratory burst activity increased in groups 1 and 2 , and by comparing the means of the three groups, no significant differences in respiratory burst activity value were seen in the different sampling days $(P>0.05)$.

\section{Discussion}

Selenium (Se), as an essential micronutrient for animals, has three levels of biological activities: (1) trace levels are
Table 2 Changes (mean $\pm \mathrm{SD})$ in respiratory burst activity of each of the groups during sampling at different days

$* P<0.05$ (significant increase on
day 0 )
day 0 )

\begin{tabular}{lccc}
\hline Group sampling days & 1 (nano-selenium) & 2 (sodium selenite) & Control \\
\hline 0 & $11.174 \pm 6.568$ & $11.566 \pm 5.325$ & $11.364 \pm 5.649$ \\
10 & $13.260 \pm 6.952$ & $16.00 \pm 6.332$ & $12.542 \pm 6.252$ \\
20 & $14.640 \pm 8.395$ & $18.864 \pm 9.099$ & $12.566 \pm 7.516$ \\
30 & $21.322 \pm 7.316^{*}$ & $24.778 \pm 10.123^{*}$ & $13.912 \pm 7.511$ \\
$P$ value & $P \leq 0.05$ & $P<0.05$ & $P>0.05$ \\
\hline
\end{tabular}


required for normal growth and development, (2) nutritional and supra-nutritional levels can be stored and homeostatic functions will be maintained, and (3) toxic levels can result in harmful effects [20]. Different sources of Se in the basal diet could improve the final weight, relative gain rate, enzymes, etc. All forms of selenium have similar abilities that allow them to increase selenoenzyme activities [6]. Se is involved in selenoprotein-type structures which include glutathione peroxidase [21], $\mathrm{P}$ selenoprotein, and $\mathrm{W}$ selenoprotein, and as selenocysteine to GSH-Px [22] and thioredoxin reductase [23]. These proteins have a protective role against oxidant materials in body cells and cause an increase in the body's cell resistance such as immunity cells against oxidative destruction $[24,25]$. The most important action of Se is its antioxidant effects [26]. Se increases the capacity of being protected from free radicals and the immunity status of the animals [27]. Researchers in the studies showed the advantages of nano-Se effects in comparison with other forms of Se; for instance, nano-Se increases the activity of GPx, TrxR, and GST more than methylselenocysteine in mice. It can be used as a potential chemopreventive agent with significant reduced risk of Se toxicity [9]. Toxicity of nano-Se is less than that of selenomethionine, which is indicated by LD50 values, acute liver injury, and short-term toxicity. GST activity increases much earlier and more severely by nano-Se in comparison with selenomethionine and selenite $[6,28]$. Huang et al. showed that the elemental Se nanoparticles have significant effects on both scavenging the free radicals and protecting DNA against oxidation, size dependently: the smaller, the better [11]. The free radical scavenging effects of the nano-Se may be increased by the interactions between nanoparticles and BSA. The supra-nutritional dose of Se may be necessary, partially as nano-Se, which can stick to the proteins in living tissue and increase the local concentrations of Se on proteins, leading to more efficient protective effects against free radicals and the subsequent anticarcinogenetic effects. Also, by the results of this study, it can also be determined that selenium nanoparticles, in comparison with sodium selenite, have better and quicker effects on chemotactic and respiratory burst activities of neutrophils. Generally, nano-Se has stronger effects on supporting the antioxidant defense systems in comparison with inorganic forms of Se. Various factors affect the chemotactic, phagocytosis, and respiratory burst activities of neutrophils such as nutrition, and between the nutrients, selenium is very important [12]. The cells' ability to decrease changes and hydrogen peroxide elimination is dependent on selenium, so selenium deficiency can cause chemotactic suppression, and this reduces neutrophils' bactericidal activity [29]. Fraga has mentioned that selenium deficiency can increase oxygen free radicals in body tissues [14], the major negative effects of which are on the consistency of biological membranes and the performance of immunity cells [30]. Heyland (2005) has suggested selenium meal consumption as a protective antioxidant activity and a way of decreasing acute disease mortality [31]. In other studies, it was observed that neutrophils' phagocytic ability was normal at the time of selenium deficiency, but its ability to kill cells was reduced [32, 33]. Zhang et al. indicated that nano-Se had a weaker interference effect than selenite on antioxidative balance in Sedeficient mice [8]. Se causes an increase in the stability of neutrophils because it acts as an antioxidant and protects body cells against oxidative hurts [13]. Antioxidants increase cell life and survival duration in blood circulation [15]. Consequently, Se deficiency decreases the value of glutathione peroxide enzyme activity and results in reducing neutrophil activity [12], and cells become sensitized to oxidative damage, causing cell death to occur faster because of oxidative materials $[21,29]$. The major results of increasing phagocytic activities of polynuclear cells, especially neutrophils, which are the first body defensive barriers (this defensive mechanism is glutathione peroxide enzyme which contains selenium and destroys the produced peroxides), are increase in oxidative pressure [34]. Also, respiratory burst, which occurred in neutrophils, can release oxygen free radicals that play a major role in pathogenesis of foreign agents. When neutrophils stick to foreign agents, the usage of oxygen will be increased hundreds of times more than before. This causes increasing activation of the NADPH oxidase enzyme on the cell surface. After neutrophils adhere to foreign agents, they will phagocyte and destroy them [35-37]. Toxic materials that are produced in neutrophils are severely destructive and cause neutrophil membrane destruction and another cell membrane [12]. Se admixtures can prevent destruction by elimination of toxic materials and free radicals and can reduce toxic material production of neutrophils and lipid peroxidation [13-15]. In this study, in the control group, there were no significant differences in the chemotactic activity value on any of the sampling days because there were no chemotactic substances such as LPS, which is the main cause of neutrophils' orientated locomotion. In groups 1 and 2, neutrophils' chemotactic activities were increased, but nano-Se was more intense on the first 10 days $(P=$ 0.035). Although chemotaxis activity was considerably decreased by sodium selenite from day 20 on $(P=0.017)$, the reduction by nano-Se was not significant from the 20th day on $(P>0.05)$. So, it is obvious that the more intensive nano-Se is caused by neutrophils' oriented locomotion, and the higher value retained for a longer period of time in comparison with sodium selenite. Respiratory burst activity is influenced by different physiological, immunological, and pathological factors. The effects of animal breed, illness history, and technical factors such as choice of data 
analysis method have also been under study [15]. Foreign agents' destruction ability of neutrophils was assayed with NBT reduction test [17]. In the present study, all sheep have similar ability in respiratory burst activity before the consumption of nano-Se and sodium selenite, but an increase was observed after consumption of selenium admixtures. This increase in both groups 1 and 2 on the 30th day in comparison with the day 0 was significant. So, according to the present results, it is clear that, to create significant increase in neutrophils' respiratory burst activity, a period of at least 20 days is required. Radostits et al. showed that selenium requires a 20 -day period in order to maximize the number of neutrophils [12]. Thus, the role of selenium in the improvement of neutrophils' performance and survival is determined and, depending on the selenium compound, this function may differ. Therefore, nano-Se increased neutrophils' numbers, neutrophils' appetence toward chemotactic substance (LPS), the ability of neutrophils to clean up, and neutrophils' insistence against oxidative stress with more intention in comparison with sodium selenite. The type of neutrophil response varies by the severity of infection. In mild bacterial infections, neutrophil number, chemotactic activity, phagocytosis, and the ability to kill the cell increase, and after recovery become normal, while in acute infections with toxic changes in neutrophils, chemotactic activity, phagocytosis, and the ability of neutrophils to kill cells are reduced [38]. Toxic neutrophils have only $25 \%$ of the healthy neutrophil activity [39].

\section{Conclusion}

Based on all the information mentioned regarding the role of selenium, it is clear that these materials are important factors in increasing the resistance and the defensive ability of the immunity system in sheep. So, Se nanoparticles have better and stronger effects on increasing chemotactic and respiratory burst activities of neutrophils in sheep than sodium selenite. Consequently, by considering other research studies that have proved that Se nanoparticles have less toxicity and more bioability than the other forms of Se, and according to the present study that showed the benefits of Se nanoparticles in comparison with sodium selenite, the use of Se nanoparticles as food supplements in animals' diet instead of other forms of selenium is recommended.

Open Access This article is distributed under the terms of the Creative Commons Attribution Noncommercial License which permits any noncommercial use, distribution, and reproduction in any medium, provided the original author(s) and source are credited.

\section{References}

1. Eisler R (2000) Selenium handbook of chemical risk assessment: health hazards to humans plants and animals, vol 3. Lewis Publishers, Boca Raton, pp 1649-1705

2. Klasing KC (1998) Comparative avian nutrition. Oxford University Press, New York

3. Rotruck JT, Pope AL, Ganther HE (1973) Selenium: biochemical role as a component of glutathione peroxidase. Science 179:585590

4. Patching SG, Gardiner PHE (1999) Recent developments in Se metabolism and chemical speciation: a review. J Trace Elem Med Biol 13:193-214

5. Whanger PD (2004) Selenium and its relationship to cancer: an update dagger. Br J Nutr 91:11-28

6. Wang H, Zhang J, Yu H (2007) Elemental selenium at nano size possesses lower toxicity without compromising the fundamental effect on selenoenzymes: comparison with selenomethionine in mice. Free Radic Biol Med 42:1524-1533

7. Xu BH, Xu ZL, Xia MS (2003) Effect of nano red elemental selenium on GPx activity of broiler chick kidney cells in vitro. Wuhan Univ J Nat Sci 8:1167-1172

8. Zhang JS, Wang HL, Yan XX, Zhang LD (2005) Comparison of short-term toxicity between nano-Se and selenite in mice. Life Sci 76:1099-1109

9. Zhang JS, Wang XF, Xu TW (2008) Elemental selenium at nano size (Nano-Se) as a potential chemopreventive agent with reduced risk of selenium toxicity: comparison with Se-methylselenocysteine in mice. Toxicol Sci 101:22-31

10. Zhang J, Wang H, Bao Y, Zhang L (2004) Nano red elemental selenium has no size effect in the induction of seleno-enzymes in both cultured cells and mice. Life Sci 75:237-244

11. Huang B, Zhang J, Hou J, Chen C (2003) Free radical scavenging efficiency of ano-Se in vitro. Free Radic Biol Med 35:805-813

12. Radostits OM, Gay CC, Hinchcliff KW, Constable PD (2007) Veterinary medicine: a textbook of the diseases of cattle, horses, sheep, pigs and goats, 10th edn. Saunders, Spain, pp $552-557$

13. Bickhardt K, Ganterm M, Sallmann P (1999) Investigation of the manifestation of vitamin $\mathrm{E}$ and selenium deficiency in sheep and goats. Dtsch-Tierarztliche-Wochenschr 106:242-247

14. Fraga CG, Ariass RF, Llesuy SF (1987) Effect of vitamin E and Se deficiency on rat liver chemiluminescence. Biochem G 242:383-392

15. Hodgson JC, Watkins CA, Bayne CW (2006) Contribution of respiratory burst activity to innate immune function and the effect of disease status and agent on chemiluminescence responses by ruminant phagocytes in vitro. Vet Immun Immunopathol 112:12-23

16. Baehner RL, Nathan DG (1968) Quantitative nitro blue tetrazolium test in chronic granulomatous disease. Nut Med 278:971-976

17. Rossi F (1986) The O2-forming NADPH oxidase of the phagocytes nature, mechanism of activation and function. Biochem Biophys J 853:65-89

18. Allen LA, Aderem A (1996) Mechanisms of phagocytosis. Current Immun 8:36-40

19. Kampen AH, Tollersrud T, Larsen S (2004) Repeatability of flow cytometric and classical measurement of phagocytosis and respiratory burst in bovine polymorphonuclear leukocytes. Vet Immun Immunopathol 97:105-114

20. Hamilton SJ (2004) Review of selenium toxicity in the aquatic food chain. Sci Total Env 326:1-31

21. Hoekstra WG (1975) Biochemical function of selenium and its relation to vitamin E. Fed Pro 34:2083-2090 
22. Segalés J, Allan GM, Domingo M (2005) Porcine circovirus diseases. Ani Health Res Rev 6:119-142

23. Yu HJ, Liu JQ, Böck A, Li J, Luo GM, Shen JC (2005) Engineering glutathione transferase to a novel glutathione peroxidase mimic with high catalytic efficiency. J Biol Chem 280:11930-11935

24. Burk RF, Hill KE, Motley AK (2003) Selenoprotein metabolism and function: evidence for more than one function for selenoprotein. J Nutr 133:1517-1520

25. Mazur A, Nasrri F, Rock E (1996) Diets deficient in selenium and vitamin $\mathrm{E}$ affect plasma lipoprotein and apolipoprotein concentrations in the rat. Br J Nutr 76:899-907

26. Levander OA, Burk RF (1994) Selenium. In: Shils ME, Olson JA, Shike M (eds) Modern nutrition in health and disease. Lea \& Febiger, Philadelphia

27. Kumar N, Garg AK, Mudgal V (2008) Effect of different levels of selenium supplementation on growth rate, nutrient utilization, blood metabolic profile, and immune response in lambs. Biol Trace Elem Res 126:44-56

28. Li H, Zhang J, Wang T, Luo W, Zhou Q, Jiang J (2008) Elemental selenium particles at nano-size (Nano-Se) are more toxic to Medaka (Oryzias latipes) as a consequence of hyper-accumulation of selenium: a comparison with sodium selenite. Aqu Toxi 89:251-256

29. Ganther HE, Serfass RE (1975) Defective microbicidal activity in glutathione peroxidase deficient neutrophils of selenium deficient rats. Nature (London) 255:640-641
30. Lessard M, Yang WC, Elliott GS (1991) Cellular immune response in pigs fed a vitamin E- and Se-deficient diet. J Anim Sci 69:1575-1582

31. Heyland DK, Dhaliwal R, Suchner U (2005) Antioxidant nutrients: a systematic review of trace elements and vitamins in the critically ill patient. Int Car Med 31:327-337

32. Boyne R, Arthur JR (1979) Alteration of neutrophil function in selenium deficient cattle. J Com Path 89:151-158

33. Ganther HE (1978) Modification of methylmercury toxicity and metabolism by selenium and vitamin E: possible mechanisms. Env Health Pers 25:71-76

34. Bernard P, Negretti DE, Bratter VE (2000) Influence of high dietary selenium intake on the thyroid hormone level in human serum. J Trace Elem Med Biol 20:163-166

35. Abbas AK, Lichtman AH (2005) Cellular and molecular immunology. Updated edition (Book + Student Consult + Evolve), 5th edn. Saunders, Philadelphia

36. Stites DP, Tristram GP, Abba IT (2001) Medical immunology, 10th edn. McGraw-Hill, New York

37. Thomas JK, Barbara AO, Richard AG (2006) Kuby immunology, 6th edn. Freeman, New York

38. Krukowski ZH, Smith G (1983) A unifying concept of polymorphonuclear neutrophil function in acute bacterial infection. Wor J Sur 7:424-429

39. McCall CE, Caves J, Cooper R (1973) Functional characteristic of human toxic neutrophils. J Inf Dis 124:68-80 